\title{
Vector boson scattering, triple gauge-boson production and limits on anomalous quartic gauge-boson couplings with the ATLAS detector
}

\author{
Hulin Wang* \\ Southern Methodist University (US) \\ E-mail: Hulin.Wang@cern.ch \\ On behalf of the ATLAS Collaboration
}

The production of vector boson scattering and triple gauge bosons are studied using $p p$ collisions at $\sqrt{s}=8 \mathrm{TeV}$ with the ATLAS detector at the Large Hadron Collider. The vector boson scattering processes of $W^{ \pm} W^{ \pm}\left(\rightarrow \ell^{ \pm} v \ell^{ \pm} v\right) j j, W Z(\rightarrow \ell v \ell \ell) j j, W W / W Z(\rightarrow \ell v j j) j j$, and exclusive $\gamma \gamma \rightarrow W^{+} W^{-}\left(\rightarrow e^{ \pm} \mu^{\mp}\right)$ are presented. The triple gauge-boson production of $W(\rightarrow \ell v) \gamma \gamma$, $Z(\rightarrow e e / \mu \mu / \nu v) \gamma \gamma$, and $W^{ \pm} W^{ \pm} W^{\mp}\left(\rightarrow \ell^{ \pm} v \ell^{ \pm} v \ell^{\mp} v / \ell^{ \pm} v \ell^{ \pm} v j j\right)$ are presented. The production cross sections are measured and compared to the predictions from the Standard Model. Modelindependent searches for new physics are studied via anomalous quartic gauge-boson couplings.

38th International Conference on High Energy Physics 3-10 August 2016

Chicago, USA

${ }^{*}$ Speaker. 


\section{Introduction}

Vector boson scattering (VBS) processes provide a unique way to probe the mechanism of electroweak symmetry breaking. In the absence of a standard model (SM) Higgs boson, the amplitudes of longitudinally polarized VBS processes increase with $\sqrt{s}$, begin to violate unitarity at $\mathrm{TeV}$ scale. The measurements of VBS production cross sections can be used to test whether the $125 \mathrm{GeV}$ SM-like Higgs boson discovered at the Large Hadron Collider (LHC) fully unitarizes the VBS scattering amplitudes.

The non-Abelian nature of the electroweak $\mathrm{SU}(2) \times \mathrm{U}(1)$ structure predicts and determines the gauge-boson self-couplings. In the SM, charged quartic gauge-boson couplings (QGCs) ( $W W W W$, $W W Z Z, W W Z \gamma, W W \gamma \gamma)$ are allowed whereas neutral QGCs (ZZZZ, ZZZ $, Z Z \gamma \gamma, Z \gamma \gamma \gamma, \gamma \gamma \gamma \gamma)$ are forbidden. Possible physics beyond the SM could affect these QGCs, and manifest itself as anomalous QGC (aQGC).

At the LHC, VBS and triple gauge-boson production are the main processes to probe the QGCs. The studies of VBS and triple gauge-boson production with the ATLAS detector [1] are described in Section 2 and 3, respectively. Section 4 provides the summary.

\section{Vector boson scattering}

2.1 $W^{ \pm} W^{ \pm}\left(\rightarrow \ell^{ \pm} v \ell^{ \pm} v\right) j j$

The first study of VBS process with ATLAS is via the $W^{ \pm} W^{ \pm}\left(\rightarrow \ell^{ \pm} v \ell^{ \pm} v\right) j j$ final state at $\sqrt{s}=8 \mathrm{TeV}$ [2], where $j$ denotes a hadronic jet. Two classes of physical processes give rise to $V V j j$ final state. The first process, which includes VBS contributions, involves exclusively weak interactions at Born level (of order $\alpha_{\mathrm{EW}}^{4}$ witihout considering the boson decay) and is referred to as electroweak production. The second process involves both the strong and electroweak interactions at Born level (of order $\alpha_{\mathrm{s}}^{2} \alpha_{\mathrm{EW}}^{2}$ ) and is referred to as strong production. Compared to other final states, the $W^{ \pm} W^{ \pm} j j$ has an advantage of having lower contribution from strong production. The observed (expected) significance for electroweak production is 3.6 (2.8) standard deviations. The measured fiducial cross section for electroweak production is $1.3 \pm 0.4$ (stat.) \pm 0.2 (syst.) fb in agreement with the SM prediction of $0.95 \pm 0.06 \mathrm{fb}$ at the next-to-leading order (NLO). This final state can be used to probe the aQGCs from the $W W W W$ vertex. The limits on the parameters inducing the $W W W W$ aQGCs are obtained.

ATLAS has updated this study [3] with improvement on the limits on the aQGCs. The expected and observed limits at 95\% confidence level (C.L.) on the aQGC parameters plane $\left(\alpha_{4}, \alpha_{5}\right)$ are shown in Figure 1.

\subsection{WZ( $\rightarrow \ell v \ell \ell) j j$}

A search for VBS process with $W Z(\rightarrow \ell v \ell \ell) j j$ final state at $\sqrt{s}=8 \mathrm{TeV}$ [4] resulted in an upper limit at 95\% C.L. on the fiducial cross section at $0.63(0.45)$ fb for observed (expected) values. The measured electroweak production cross section is $0.29_{-0.12}^{+0.14}(\mathrm{stat})_{-0.10}^{+0.09}$ (syst.) fb and is consistent with the SM prediction of $0.13 \pm 0.01 \mathrm{fb}$ at NLO level. These final states can be used to probe the aQGCs from the $W W Z Z$ vertex. The limits on the $\alpha_{4}$ and $\alpha_{5}$ parameters inducing the 
WWZZ aQGCs are shown in Figure 1 as the expected and observed two-dimensional contours at 95\% C.L.

\section{$2.3 W W / W Z(\rightarrow \ell v j j) j j$}

ATLAS recently performed a search for anomalous electroweak production of $W W / W Z(\rightarrow$ $\ell v j j) j j$ at $\sqrt{s}=8 \mathrm{TeV}$ [5]. The large background makes it a challenging final state to measure the SM signal. On other hand, compared with the fully leptonic final states, it has more sensitivity to the aQGCs due to larger branch fraction and one less neutrino in the final state to complicate the kinematic reconstruction. Two selection criteria are used to optimize the selection efficiency of $V \rightarrow j j$. A "resolved" selection is used to select the events where $V \rightarrow j j$ is reconstructed as two separate small-radius jets. A "merged" selection is developed to identify events where $V \rightarrow j j$ is reconstructed as a single large-radius jet. It significantly improves the aQGC sensitivity because the aQGCs tend to have larger $m_{\mathrm{T}}(W V)$ compared with the SM, where the events are more likely to fall into the "merged" category than the events with smaller $m_{\mathrm{T}}(W V)$. The two-dimensinal (2D) confidence region for $\alpha_{4}$ and $\alpha_{5}$ is shown in Figure 1.

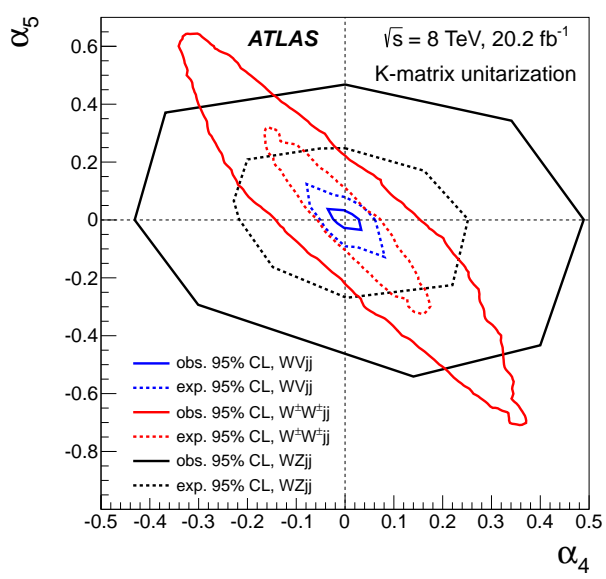

Figure 1: Results of the search for anomalous electroweak production of $W W / W Z(\rightarrow \ell v j j) j j$ at $\sqrt{s}=8$ TeV with ATLAS [5]. The observed 2D confidence region (solid blue contour) for $\alpha_{4}$ and $\alpha_{5}$, at 95\% C.L. The expected 2D confidence region (dotted blue contour) is also shown, computed using the Asimov dataset. Results from $W W / W Z(\rightarrow \ell v j j)$ VBS analysis [5] (in blue) are compared to observed and expected confidence regions from previous ATLAS analyses of $W^{ \pm} W^{ \pm}$[3] (in red) and $W Z$ [4] (in black) VBS production.

\section{$2.4 \gamma \gamma \rightarrow W^{+} W^{-}\left(\rightarrow e^{ \pm} \mu^{\mp}\right)$}

Measurements of exclusively produced $W$ boson pairs in the process of $\gamma \gamma \rightarrow W^{+} W^{-}$has been performed using $e^{ \pm} \mu^{\mp}$ final states at $\sqrt{s}=8 \mathrm{TeV}$ with ATLAS [6]. The exclusive $W^{+} W^{-}$events are produced when each proton emits a photon and the two photons annihilate, either via $t$ - and $u$ channel $W$-exchange diagrams involving trilinear gauge-boson couplings or via the $W W \gamma \gamma$ QGCs, to create a $W^{+} W^{-}$pair. Exclusive candidates are characterized by an absence of tracks, other than the tracks from $W^{+} W^{-}$pair decay products. In order to distinguish the exclusive events from the inclusive events, it is required that no additional track comes from the vertex of the two leptons 
from the $W^{+} W^{-}$pair decay. Figure 2 shows the $p_{\mathrm{T}}^{e \mu}$ and $\Delta \phi_{e \mu}$ distributions of the $e \mu$ pairs in the signal region. The observed signal significance is 3.0 standard deviations. The measured cross section extrapolated to the full phase space is $6.9 \pm 2.2$ (stat.) \pm 1.4 (syst.) fb, to be compared to the SM prediction of $4.4 \pm 0.3 \mathrm{fb}$ at NLO level. The limits on the parameters inducing the $W W \gamma \gamma$ aQGCs are also extracted.
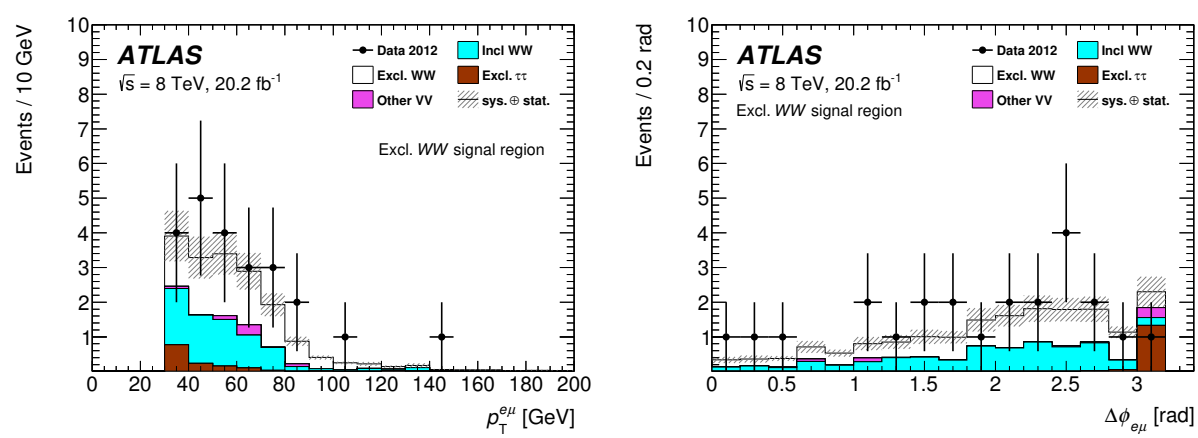

Figure 2: Results of the measurements of exclusively produced $W$ boson pairs in the process of $\gamma \gamma \rightarrow$ $W^{+} W^{-}\left(\rightarrow e^{ \pm} \mu^{\mp}\right)$ at $\sqrt{s}=8 \mathrm{TeV}$ with ATLAS [6]. The $p_{\mathrm{T}}^{e \mu}$ (left) and $\Delta \phi_{e \mu}$ (right) distributions in the exclusive $W^{+} W^{-}$signal region. The inclusive $W^{+} W^{-}$estimate includes small contributions from other backgrounds (Drell-Yan, $W+$ jets, and top-quark production).

\section{Triple gauge-boson production}

3.1 $W(\rightarrow \ell v) \gamma \gamma$

The first study of triple gauge-boson production with ATLAS has been done using the $W(\rightarrow$ $\ell v) \gamma \gamma$ final state at $\sqrt{s}=8 \mathrm{TeV}$ [7]. The measurements are performed in the inclusive $\left(N_{\text {jets }} \geq\right.$ $0)$ and exclusive $\left(N_{\text {jets }}=0\right)$ regions. The observed signal significance is more than 3 standard deviations in the inclusive region. Table 1 shows the measured cross sections in the fiducial region and the SM predictions at NLO level. The limits on the parameters inducing the $W W \gamma \gamma$ aQGCs are also calculated.

\begin{tabular}{|c|c|c|}
\hline & $\overline{\sigma^{\text {fid }}[\mathrm{fb}]}$ & 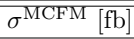 \\
\hline \multicolumn{3}{|c|}{ Inclusive $\left(N_{\text {jet }} \geq 0\right)$} \\
\hline $\begin{array}{l}\mu \nu \gamma \gamma \\
e \nu \gamma \gamma \\
\ell \nu \gamma \gamma\end{array}$ & 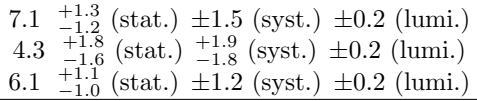 & $2.90 \pm 0.16$ \\
\hline \multicolumn{3}{|c|}{ Exclusive $\left(N_{\text {jet }}=0\right)$} \\
\hline $\begin{array}{l}\mu \nu \gamma \gamma \\
e \nu \gamma \gamma \\
\ell \nu \gamma \gamma\end{array}$ & 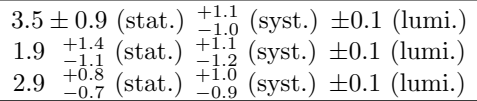 & $1.88 \pm 0.20$ \\
\hline
\end{tabular}

Table 1: Results of $W(\rightarrow \ell v) \gamma \gamma$ production measurements at $\sqrt{s}=8 \mathrm{TeV}$ with ATLAS [7]. Measurements of the $W(\rightarrow \ell v) \gamma \gamma$ inclusive and exclusive fiducial cross sections. 


\section{$3.2 Z(\rightarrow e e / \mu \mu / \nu v) \gamma \gamma$}

ATLAS performed the measurements of $Z(\rightarrow e e / \mu \mu / v v) \gamma \gamma$ production at $\sqrt{s}=8 \mathrm{TeV}$ [8]. The measurements are performed in the inclusive $\left(N_{\text {jets }} \geq 0\right)$ and exclusive $\left(N_{\text {jets }}=0\right)$ regions. The observed signal significance of $Z(\rightarrow e e / \mu \mu) \gamma \gamma$ is 6.3 (6.0) standard deviations for inclusive (exclusive) regions. Table 2 shows the measured cross sections in the extended fiducial regions and the SM predictions at NLO level. This final state can be used to probe the aQGCs from the $Z \gamma \gamma \gamma$ and $Z Z \gamma \gamma$ vertices. Constrains on the relevant parameters are reported.

\begin{tabular}{|c|c|c|}
\hline Channel & Measurement $[\mathrm{fb}]$ & MCFM Prediction [fb] \\
\hline \multicolumn{3}{|c|}{$N_{\text {jets }} \geq 0$} \\
\hline$e^{+} e^{-} \gamma \gamma$ & $6.2_{-1.1}^{+1.2}$ (stat.) \pm 0.4 (syst.) \pm 0.1 (lumi.) & \multirow{3}{*}{$3.70_{-0.11}^{+0.21}$} \\
\hline$\mu^{+} \mu^{-} \gamma \gamma$ & $3.83_{-0.85}^{+0.95}$ (stat. $)_{-0.47}^{+0.48}$ (syst.) \pm 0.07 (lumi.) & \\
\hline$\ell^{+} \ell^{-} \gamma \gamma$ & $5.07_{-0.68}^{+0.73}$ (stat. $)_{-0.38}^{+0.41}$ (syst.) \pm 0.10 (lumi.) & \\
\hline$v \bar{v} \gamma \gamma$ & $2.5_{-0.9}^{+1.0}$ (stat.) \pm 1.1 (syst.) \pm 0.1 (lumi. $)$ & $0.737_{-0.032}^{+0.039}$ \\
\hline \multicolumn{3}{|c|}{$N_{\text {jets }}=0$} \\
\hline$e^{+} e^{-} \gamma \gamma$ & $4.6_{-0.9}^{+1.0}$ (stat. $)_{-0.3}^{+0.4}$ (syst.) \pm 0.1 (lumi.) & \multirow{3}{*}{$2.91_{-0.12}^{+0.23}$} \\
\hline$\mu^{+} \mu^{-} \gamma \gamma$ & $2.38_{-0.67}^{+0.77}$ (stat. $)_{-0.32}^{+0.33}$ (syst. $)_{-0.04}^{+0.05}$ (lumi. $)$ & \\
\hline$\ell^{+} \ell^{-} \gamma \gamma$ & $3.48_{-0.56}^{+0.61}$ (stat. $)_{-0.25}^{+0.29}$ (syst.) \pm 0.07 (lumi.) & \\
\hline$v \bar{v} \gamma \gamma$ & $1.18_{-0.44}^{+0.52}$ (stat.) ${ }_{-0.49}^{+0.48}$ (syst.) \pm 0.02 (lumi.) & $0.395_{-0.037}^{+0.049}$ \\
\hline
\end{tabular}

Table 2: Results of $Z(\rightarrow e e / \mu \mu / v v) \gamma \gamma$ production measurements at $\sqrt{s}=8 \mathrm{TeV}$ with ATLAS [8]. Measured and predicted cross sections for the $Z \gamma \gamma$ processes in the extended fiducial regions.

\section{3 $W^{ \pm} W^{ \pm} W^{\mp}\left(\rightarrow \ell^{ \pm} v \ell^{ \pm} v \ell^{\mp} v / \ell^{ \pm} v \ell^{ \pm} v j j\right)$}

ATLAS recently performed a search for triboson $W^{ \pm} W^{ \pm} W^{\mp}$ production in two decay channels [9]. The decay $W^{ \pm} W^{ \pm} W^{\mp} \rightarrow \ell^{ \pm} v \ell^{ \pm} v \ell^{\mp} v$ has lower branching fraction but smaller background than $W^{ \pm} W^{ \pm} W^{\mp} \rightarrow \ell^{ \pm} v \ell^{ \pm} v j j$ which has larger branching fraction but larger background. The $m_{\mathrm{T}}^{3 \ell}$ distribution for the $\ell v \ell v \ell v$ final state and the $\sum p_{\mathrm{T}}$ distribution for the $\ell \nu \ell v j j$ final state in the signal regions are shown in Figure 3. The observed (expected) significance of the signal is 0.96 (1.05) standard deviation for the combination of the two channels. Limits on the cross sections at $95 \%$ C.L. are also derived. aQGCs from the $W W W W$ vertex can be studied via this final state. The distributions from aQGCs contributions are also shown in Figure 3. Limits are set on aQGC parameters.

\section{Summary}

Studies of VBS and triple gauge-boson production with the ATLAS detector at $\sqrt{s}=8 \mathrm{TeV}$ are reported. Several final states observe a signal significance of more than 3 standard deviations. No significant deviations from the SM are observed. Constraints are derived on the parameters inducing the aQGCs.

\section{References}

[1] ATLAS Collaboration, The ATLAS Experiment at the CERN Large Hadron Collider, JINST 3, S08003 (2008). 

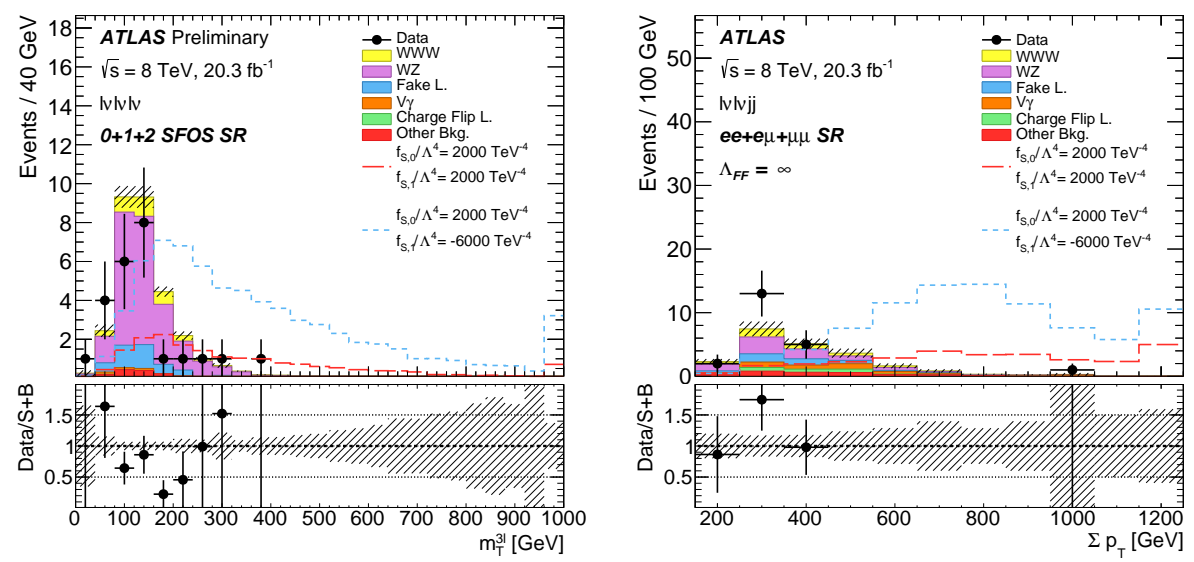

Figure 3: Results of the search for triboson $W^{ \pm} W^{ \pm} W^{\mp}\left(\rightarrow \ell^{ \pm} v \ell^{ \pm} v \ell^{\mp} v / \ell^{ \pm} v \ell^{ \pm} v j j\right)$ production at $\sqrt{s}=8$ TeV with ATLAS [9]. The distribution of $m_{\mathrm{T}}^{3 \ell}$ for the $\ell v \ell v \ell v$ channel (left) and the distribution of $\sum p_{\mathrm{T}}$ for the $\ell v \ell v j j$ channel (right) as observed in the data (dots with error bars indicating the statistical uncertainties) and as expected from SM signal and background processes. The ratios between the observed numbers of events in data and the expected SM signal plus background contributions are shown in the lower panels. The hashed bands result from the systematic uncertainties on the sum of the signal plus background contributions. Contributions from aQGCs are also shown, assuming the non-unitarized case $\left(\Lambda_{\mathrm{FF}}=\infty\right)$ and two different sets of $f_{S, 0} / \Lambda^{4}$ and $f_{S, 1} / \Lambda^{4}$ configurations $\left(f_{S, 0} / \Lambda^{4}=2000 \mathrm{TeV}^{-4}, f_{S, 1} / \Lambda^{4}=2000 \mathrm{TeV}^{-4}\right.$ and $f_{S, 0} / \Lambda^{4}=$ $\left.2000 \mathrm{TeV}^{-4}, f_{S, 1} / \Lambda^{4}=-6000 \mathrm{TeV}^{-4}\right)$.

[2] ATLAS Collaboration, Evidence for Electroweak Production of $W^{ \pm} W^{ \pm} j$ in pp Collisions at $\sqrt{s}=8$ TeV with the ATLAS Detector, Phys. Rev. Lett. 113, 141803 (2014) [arXiv: 1405 . 6241 [hep-ex]].

[3] ATLAS Collaboration, Measurement of $W^{ \pm} W^{ \pm}$Vector Boson Scattering and Limits on Anomalous Quartic Gauge Couplings with the ATLAS Detector, CERN-EP-2016-167. Paper to be submitted.

[4] ATLAS Collaboration, Measurements of $W^{ \pm} Z$ production cross sections in pp collisions at $\sqrt{s}=8$ TeV with the ATLAS detector and limits on anomalous gauge boson self-couplings, Phys. Rev. D 93, 092004 (2016) [arXiv:1603.02151 [hep-ex]].

[5] ATLAS Collaboration, Search for anomalous electroweak production of $W W / W Z$ in association with a high-mass dijet system in pp collisions at $\sqrt{s}=8 \mathrm{TeV}$ with the ATLAS detector, Submitted to Phys. Rev. $D$ [arXiv:1609.05122 [hep-ex]].

[6] ATLAS Collaboration, Measurement of exclusive $\gamma \gamma \rightarrow W^{+} W^{-}$production and search for exclusive Higgs boson production in pp collisions at $\sqrt{s}=8 \mathrm{TeV}$ using the ATLAS detector, Phys. Rev. D 94, 032011 (2016) [arXiv:1607.03745 [hep-ex]].

[7] ATLAS Collaboration, Evidence of $W \gamma \gamma$ Production in pp Collisions at $\sqrt{s}=8 \mathrm{TeV}$ and Limits on Anomalous Quartic Gauge Couplings with the ATLAS Detector, Phys. Rev. Lett. 115, 031802 (2015) [arXiv:1503.03243 [hep-ex]].

[8] ATLAS Collaboration, Measurements of Z $\gamma$ and $Z \gamma \gamma$ production in pp collisions at $\sqrt{s}=8$ TeV with the ATLAS detector, Phys. Rev. D 93, 112002 (2016) [arXiv:1604.05232 [hep-ex]].

[9] ATLAS Collaboration, Search for triboson $W^{ \pm} W^{ \pm} W^{\mp}$ production in pp collisions at $\sqrt{s}=8 \mathrm{TeV}$ with the ATLAS detector, Submitted to Eur. Phys. J. C [arXiv:1610.05088 [hep-ex]]. 